\title{
Suplementação com vitamina d em mulheres pós-menopáusicas: uma análise das publicações científicas atuais
}

Vitamin d supplementation in postmenopausal women: an analysis of current scientific publications

Suplementos de vitamina d en mujeres posmenopáusicas: un análisis de las publicaciones científicas actuales

Rebeca Muálem de Moraes Santos

ORCID: https://orcid.org/0000-0002-8196-5706 Instituto de Educação Superior do Vale do Paraíba, Brasil E-mail: rebecamualem@hotmail.com

Vitória Fonseca Viana

ORCID: https://orcid.org/0000-0001-5764-7073 Instituto de Educação Superior do Vale do Paraíba, Brasil E-mail: vivfonseca27@gmail.com

Yarah Pereira Rodrigues Véras ORCID: https://orcid.org/0000-0001-9978-2580 Instituto de Educação Superior do Vale do Paraíba, Brasil E-mail: yarahprodrigues@icloud.com

Débora Karine dos Santos Pacífico

ORCID: https://orcid.org/0000-0002-2505-692X Instituto de Educação Superior do Vale do Paraíba, Brasil E-mail: deborakrine02@gmail.com

Francisco David de Souza e Silva

ORCID: https://orcid.org/ 0000-0002-0883-4144 Instituto de Educação Superior do Vale do Paraíba, Brasil

E-mail: franciscoDavid2016@outlook.com

Myrella Antonia Almeida Cunha

ORCID: https://orcid.org/0000-0003-1103-9798 Instituto de Educação Superior do Vale do Paraíba, Brasil E-mail: myrellacunhamed@gmail.com

Fabbio Ronnyel Rodrigues Baldoino

ORCID: https://orcid.org/0000-0003-4393-6039 Instituto de Educação Superior do Vale do Paraíba, Brasil E-mail: fabbiobald@gmail.com

Maria Jayanne dos Santos Benício ORCID: https://orcid.org/0000-0002-3157-909X Instituto de Educação Superior do Vale do Paraíba, Brasil E-mail: jayannebenicio@gmail.com

Pedro Jackson dos Santos Benício ORCID: https://orcid.org/0000-0002-9880-3041 Instituto de Educação Superior do Vale do Paraíba, Brasil

E-mail: pedrojacksonbenicio@gmail.com

Hellen Pereira Melo

ORCID: https://orcid.org/0000-0003-1813-7577 Instituto de Educação Superior do Vale do Paraíba, Brasil E-mail: hellen_melo04@hotmail.com

José de Ribamar Ramos Neto

ORCID: https://orcid.org/0000-0002-4284-0070 Instituto de Educação Superior do Vale do Paraíba, Brasil E-mail: ramosneto14@gmail.com Eduarda Viana Trajano

ORCID: https://orcid.org/0000-0002-7457-0431 Instituto de Educação Superior do Vale do Paraíba, Brasil

E-mail: eduarda_trajano13@hotmail.com

Gabriela Dantas Carvalho

ORCID: https://orcid.org/0000-0002-9571-3323

Universidade Federal do Delta do Parnaíba, Brasil

E-mail: ftgabrieladantas@hotmail.com

Thiago de Souza Lopes Araújo ORCID: https://orcid.org/0000-0001-8543-8498 


\begin{abstract}
Resumo
A menopausa é definida como a interrupção permanente do ciclo menstrual, sendo diagnosticada após um período de 12 meses consecutivos de amenorreia. Mulheres na pós-menopausa, os níveis de vitamina D podem ser alterados. Desta forma, alguns estudos têm sugerido a suplementação exógena desse nutriente como alternativa terapêutica. $\mathrm{O}$ objetivo foi realizar uma revisão de literatura sobre a suplementação exógena isolada e/ou combinada de vitamina D em mulheres na pós-menopausa. Reuniu-se diversos artigos publicados entre 2016 e 2020. Utilizou-se como descritores: "pós-menopausa", "suplementação nutricional" e "vitamina D", definidos conforme plataforma dos Descritores em Ciências da Saúde - DeCS. As bases de dados utilizadas foram: Scielo, LILACS e PUBMED. A seleção dos artigos seguiu critérios de inclusão e exclusão, previamente estabelecidos. Um total de 21 artigos foi incluído no estudo, sendo a maior parte deles publicada em 2018 ( $\mathrm{n}=9,42,85 \%)$. Os estudos selecionados avaliaram os efeitos desta suplementação em diferentes aspectos. Comparou-se as altas e baixas doses da suplementação, analisou-se os efeitos de uma combinação da vitamina D com outros nutrientes, bem como a influência de um processo educativo sobre o tema com as participantes. Estas mulheres foram avaliadas quanto à tal terapia, considerando a prática de atividade física, o perfil sérico de hormônios sexuais, o risco de câncer em fumantes, bem como quanto aos parâmetros musculares e inflamatórios. Diante do exposto, observou-se a suplementação exógena com vitamina D é uma opção terapêutica em constante avaliação. Foi constatado ainda que a suplementação combinada foi a abordagem mais desenvolvida entre os estudos.
\end{abstract}

Palavras-chave: Pós-menopausa; Suplementação nutricional; Vitamina D.

\begin{abstract}
Menopause is defined as the permanent interruption of the menstrual cycle, being diagnosed after a period of 12 consecutive months of amenorrhea. In these women, vitamin D levels can be changed. Thus, some studies have suggested the exogenous supplementation of this nutrient as a therapeutic alternative. The objective was to conduct a literature review on exogenous isolated and/or combined vitamin D supplementation in postmenopausal women. Several articles published between 2016 and 2020 were collected. The following descriptors were used: "postmenopause", "nutritional supplementation" and "vitamin D", defined according to the Health Sciences Descriptors DeCS platform. The databases used were Scielo, LILACS and PUBMED. The selection of articles followed inclusion and exclusion criteria, previously established. 21 articles were included in the study, most of which were published in $2018(\mathrm{n}=9.42 .85 \%)$. The selected studies evaluated the effects of this supplementation in different aspects. The high and low doses of supplementation were compared, the effects of a combination of vitamin D with other nutrients were analyzed, as well as the influence of an educational process on the topic with the participants. These women were evaluated for such therapy, considering the practice of physical activity, the serum profile of sex hormones, the risk of cancer in smokers, as well as muscle and inflammatory parameters. In view of the above, it was observed that exogenous supplementation with vitamin D is a therapeutic option under constant evaluation. It was also found that combined supplementation was the most developed approach among studies.
\end{abstract}

Keywords: Post-menopause; Nutritional supplementation; D vitamin.

\title{
Resumen
}

La menopausia se define como la interrupción permanente del ciclo menstrual, siendo diagnosticada luego de un período de 12 meses consecutivos de amenorrea. En estas mujeres, los niveles de vitamina D pueden modificarse. Así, algunos estudios han sugerido la suplementación exógena de este nutriente como alternativa terapéutica. El objetivo fue realizar una revisión de la literatura sobre la suplementación con vitamina D exógena aislada y/o combinada en mujeres posmenopáusicas. Se recogieron varios artículos publicados entre 2016 y 2020. Se utilizaron los siguientes descriptores: "posmenopausia", "suplementación nutricional" y "vitamina D", definidos según la plataforma Health Sciences Descriptors - DeCS. Las bases de datos utilizadas fueron: Scielo, LILACS y PUBMED. La selección de artículos siguió los criterios de inclusión y exclusión previamente establecidos. En el estudio se incluyeron un total de 21 artículos, la mayoría de los cuales fueron publicados en 2018 ( $n=9,42,85 \%)$. Los estudios seleccionados evaluaron los efectos de esta suplementación en diferentes aspectos. Se compararon las dosis altas y bajas de suplementación, se analizaron los efectos de una combinación de vitamina D con otros nutrientes, así como la influencia de un proceso educativo sobre el tema con los participantes. Estas mujeres fueron evaluadas para dicha terapia, considerando la práctica de actividad física, el perfil sérico de hormonas sexuales, el riesgo de cáncer en fumadores, así como parámetros musculares e inflamatorios. En vista de lo anterior, se observó que la suplementación exógena con vitamina D es una opción terapéutica en constante evaluación. También se encontró que la suplementación combinada fue el enfoque más desarrollado entre los estudios. 
Palabras clave: Posmenopausia; Suplementación nutricional; Vitamina D.

\section{Introdução}

De acordo com o Ministério da Saúde, a menopausa é definida como a interrupção permanente do ciclo menstrual, com o diagnóstico realizado de forma retroativa, após um período de 12 meses consecutivos de amenorreia. O climatério como etapa do ciclo de vida, promove a menopausa, geralmente, entre os 48 e 50 anos de idade. Em outros casos, a menopausa pode acontecer de maneira precoce, antes dos 40 anos, sendo conhecida como falência ovariana precoce (Brasil, 2016).

Fisiologicamente, embora ocorra uma influência do eixo hipotálamo hipofisário, a menopausa natural pode ser considerada como um evento ovariano, secundário à atresia fisiológica dos folículos primordiais (Lui Filho et al 2015). O climáterio é conhecida por envolver alterações em níveis hormonais, mudanças fisiológicas e psicossociais, que afetam consideravelmente a vida pessoal, social e profissional das mulheres, incluindo episódios de calor intenso e suores noturnos, insônia e instabilidade de humor (Monteleone et al., 2018; Khoudary et al., 2019).

O diagnóstico de tais alterações podem ser realizado sem exames laboratoriais, utilizando para este fim, as manifestações clínicas características do quadro (Brasil, 2016). Neste sentido, considera-se que mulheres com idade acima de 45 anos, estejam no período conhecido como perimenopausa, onde se observa a presença de sintomas vasomotores com menstruação irregular. Por outro lado, aquelas mulheres que não menstruam há mais de 12 meses estão na pós-menopausa, propriamente dita (NICE, 2015; Potter et al., 2018).

Durante a perimenopausa e a transição da menopausa, as mulheres experimentam muitas mudanças. No início da perimenopausa, algumas delas vivenciam uma variação na duração dos ciclos menstruais, enquanto que em um estágio tardio desta fase, a maior parte das mulheres terá episódios de amenorreia superior a 60 dias, que pode durar até três anos. Durante a transição da perimenopausa e por até 2 anos após a menopausa, os níveis do hormônio folículo-estimulante estão elevados, enquanto o estradiol (E2) está diminuindo (Harlow et al., 2012; Potter et al., 2018).

$\mathrm{Na}$ fase da pós-menopausa, uma das importantes mudanças no organismo feminino corresponde às alterações nos níveis de vitamina D. A diminuição deste nutriente é considerada um problema de saúde pública, seja no climatério, seja em outras patologias, tendo em vista a alta prevalência mundial. Além disso, tal redução pode ainda contribuir para a ocorrência de inúmeras doenças agudas e crônicas, incluindo o câncer, deficiência cognitiva, diabetes e doenças cardiovasculares (PérezLópez et al., 2012; Lerchbaum, 2014; Pérez-López; Chedraui \& Pilz, 2020).

A vitamina D é conhecida por desempenhar um importante papel na manutenção da homeostase de outros nutrientes essenciais ao organismo, como o cálcio e o fósforo, além de participar na promoção da mineralização óssea. Nesse sentido, entende-se a importância de níveis suficientes de vitamina D para a funcionalidade do corpo (Lerchbaum, 2014).

Metabolicamente, a vitamina D ocorre sob duas formas principais: vitamina D2 ou ergocalciferol, como também é conhecida, a qual é sintetizada na epiderme pela ação da radiação ultravioleta da luz solar; e na forma de vitamina D3, ou colecalciferol, formada a partir do colesterol. Ambas as formas, quando produzidas, encontram-se ainda inativas, passando então por um processo de ativação, a nível de fígado e rim, mediante a adição de grupos hidroxila, o que resulta na forma hormonal ativa, o 1 1,25-diiidroxi-vitamina D ou calcitriol (Barral; Barros \& Araújo, 2007; Castro, 2011).

Em mulheres na pós-menopausa, relata-se a ocorrência de uma alta prevalência de doenças relacionadas à vitamina $\mathrm{D}$, especificamente com alterações no metabolismo deste nutriente, como uma diminuição da síntese cutânea de vitamina D ou modificações na composição corporal, que são relevantes para a fisiologia e estado geral do nutriente (Pérez-López; Chedraui \& Pilz, 2020). Desta maneira, compreende-se que a menopausa e a deficiência de vitamina D são problemas intimamente relacionados, e que proporcionam diversos resultados adversos à saúde da mulher, o que inclui a perda óssea, distúrbios do 
humor, associação com a síndrome metabólica, aumento do risco de doenças cardiovasculares e câncer (Lerchbaum, 2014; Schmitt et al., 2017; Lee; Kim, 2018).

Partindo desta premissa, muitos estudos têm avaliado os efeitos da suplementação com vitamina $\mathrm{D}$, ou até mesmo dos seus precursores, tanto em mulheres pós menopausa como em adultos jovens (Pérez-López et al., 2012; Oliveri et al 2015; Vaes et al. 2017), tendo demonstrado efeitos promissores, tais como a proteção óssea pela reabsorção de cálcio e reposição hormonal, permitindo a síntese de hormônios que retardam o envelhecimento dos ovários . Neste sentido, a atualização constante acerca dessa temática é de grande interesse, podendo-se assegurar a compreensão e a disseminação do conhecimento sobre o assunto. Assim, o objetivo do presente estudo foi realizar uma revisão de literatura sobre a suplementação exógena isolada e/ou combinada de vitamina D em mulheres pós-menopausa.

\section{Metodologia}

No presente estudo, realizou-se uma revisão de literatura, reunindo diversas publicações científicas disponibilizadas em diferentes bases de dados, nos últimos cinco anos (2016-2020). Para a elaboração deste trabalho, foram consideradas artigos científicos relacionados ao tema, que pudessem nortear a investigação.

As revisões bibliográficas podem ser consideradas uma forma de pesquisa, utilizando-se de resultados de outros estudos para promover uma discussão sobre o referido tema, com uma análise das mais distintas e recentes publicações (Rother, 2007; Prodanov \& Freitas, 2013). As bases para a revisão da literatura, incluem artigos científicos publicados em periódicos nacionais e internacionais, bem como livros, monografias, dissertações e teses (Prodanov, 2013). No presente estudo elaborou-se uma revisão do tipo integrativa. Esse tipo de revisão bibliográfica é considerada uma importante contribuição acadêmica, pois resume de maneira sistemática, ordenada e abrangente, os resultados alcançados em diversos estudos (Ercole, Melo \& Alcoforado, 2014).

Para a elaboração deste estudo, inicialmente delimitou-se o tema e a questão norteadora: o papel da vitamina D nas alterações pós-menopausa. Em seguida, os principais descritores, ou seja, as palavras-chave, empregados na busca das publicações foram definidos. Os descritores foram estabelecidos de acordo plataforma dos Descritores em Ciências da Saúde (DeCS), correspondendo assim as seguintes palavras-chave: "pós-menopausa", "suplementação nutricional" e "vitamina D", de forma isolada ou combinada por meio dos operadores boleandos - $A N D$ ou - $O \mathrm{R}$. Tais descritores também foram utilizados em inglês para a busca de publicações internacionais. Em seguida, determinou-se as bases de dados para coleta das publicações, sendo elas: Scielo (Scientific Electronic Library Online), LILACS (Literatura Latino Americana e do Caribe em Ciências da Saúde) e PUBMED (Medical Publisher/National Library of Medicine).

A fim de garantir apenas a análise de publicações que apresentassem íntima relação com o tema, empregou-se como critérios de inclusão as publicações dentro do período selecionado, artigos completos publicados em português, inglês ou espanhol. As publicações de resumos simples e/ou expandidos, artigos que não apresentassem o tipo dados desejados, bem como outras revisões da literatura, meta-análises e além de artigos duplicados, foram excluídos da seleção. Ao final, realizouse a avaliação das publicações, a interpretação e a síntese das informações obtidas e em seguida, estas são apresentadas de maneira descritiva, com o objetivo de reunir as ideias acerca do tema proposto pelo presente estudo.

\section{Resultados e Discussão}

Dada a necessidade de atualizações no conhecimento sobre um determinado tema e considerando o objetivo do presente estudo, um total de 21 publicações foram selecionadas para análise e compreensão acerca da suplementação de 
vitamina D em mulheres na pós- menopausa. Com isso, foi possível avaliar as informações apresentadas em diferentes estudos e compreender os efeitos desta alternativa terapêutica.

Ao considerar os anos das publicações selecionadas, foi possível observar que o ano de 2018 apresentou o maior número de publicações, com $9(42,85)$ estudos publicados, conforme apresentado na Tabela 1.

Tabela 1: Quantidade de estudos incluídos no presente estudo, por ano de publicação.

\begin{tabular}{cc}
\hline Ano & $\begin{array}{c}\text { Quantidade de Publicações } \\
\text { n (\%) }\end{array}$ \\
\hline 2016 & $3(14,30)$ \\
2017 & $5(23,81)$ \\
2018 & $9(42,85)$ \\
2019 & $02(9,52)$ \\
2020 & $02(9,52)$ \\
\hline Total & $21(100 \%)$ \\
\hline
\end{tabular}

Fonte: Autores (2021).

Como relatado anteriormente, a suplementação com vitamina D tem sido utilizada como alternativa para mulheres na menopausa, uma vez há uma redução acentuada deste micronutriente nesta etapa da vida feminina (Lerchbaum, 2014; PérezLópez; Chedraui \& Pilz, 2020). Assim, considerando a possibilidade da ocorrência de outras doenças, é de extrema importância que novas alternativas terapêuticas sejam avaliadas, como é o caso da suplementação exógena com a vitamina D.

No geral, as publicações incluídas no presente estudo, avaliaram os efeitos desta suplementação em diferentes aspectos. A exemplo disso, a comparação entre altas e baixas doses da suplementação, a combinação da vitamina D com outros nutrientes, bem como a influência de um processo educativo sobre o tema com essas mulheres, foram abordagens tratadas nos estudos selecionados (Apaydin et al., 2018; Lee et al, 2019; Argyrou et al., 2020). Diante disso, no intuito de integrar as informações que compuseram a reflexão aqui exposta, a Tabela 2 apresenta os títulos, o ano de publicação e a autoria, além dos objetivos e dos principais resultados obtidos por tais estudos. 
Tabela 2: Publicações científicas sobre a suplementação exógena isolada e/ou combinada de Vitamina D em mulheres pós-menopáusicas.

Título/Autoria/Ano

Effects of vitamin D supplementation

on pulmonary function in

postmenopausal women following an aquatic exercise program./Nolasco et al., 2016.

Effect of isolated vitamin D supplementation on the rate of falls and postural balance in postmenopausal women fallers: a randomized, double-

blind, placebo-controlled trial./Cangussu et al., 2016.

Effects of vitamin D supplementation during weight loss on sex hormones in postmenopausal women./Mason et al. 2016. de vitamina D3 nos níveis séricos dos hormônios sexuais em mulheres na pósmenopausa, seguindo uma dieta de 12 meses e executando um programa de exercícios para perda de peso.

Objetivo

Resultados

Avaliar os efeitos da suplementação de pulmonar em mulheres na pós-menopausa.

Avaliar o efeito da suplementação isolada de vitamina $D$ na taxa de quedas e equilíbrio postural em mulheres pós-menopáusicas.

Serum 25-hydroxyvitamin D levels are associated with functional capacity but not with postural balance in osteoporotic post
Avaliar a associação entre os níveis de vitamina D e força muscular, equilíbrio postural e mobilidade funcional em mulheres na pósmenopausa com diagnóstico de osteoporose.
Após seis meses de intervenção, os autores observaram que o nível de 25-hidroxivitamina D (25 (OH) D), o pico de fluxo expiratório e a capacidade vital de força, apresentaram elevação no grupo de mulheres na menopausa que receberam suplementação com vitamina $\mathrm{D}$ e cálcio, e que não fizeram exercícios; bem como no grupo que recebeu a mesma suplementação e realizou exercícios aquáticos. Por tais achados, os autores concluem que a suplementação de vitamina D melhora os parâmetros de função pulmonar em mulheres na pós-menopausa.

Observou-se que houve um aumento nos níveis de vitamina $\mathrm{D}$ de $15,0 \mathrm{ng} / \mathrm{mL}$ para 27,5 ng/mL, no grupo que recebeu a suplementação com o micronutriente, durante nove meses. Além disso, o analisarem o risco de queda, os autores observaram que houve um risco maior de 2,8 para quedas recorrentes no grupo placebo, quando comparado ao grupo da suplementação. O grupo que recebeu vitamina D apresentou uma redução significativa nas oscilações látero-laterais $(35,5 \%)$ e ântero-posterior $(37,0 \%)$ do corpo, o que promoveu uma melhor postura corporal, com reflexo no menor risco de quedas.

Foi verificado um aumento expressivo nos níveis séricos de 25 -hidroxivitamina $\mathrm{D}$, e esse evento foi associado a um aumento na globulina de ligação aos hormônios sexuais, bem como a diminuições significativas nos níveis de estradiol livre e biodisponível. Os autores concluem que após 12 meses de intervenção, as mudanças no hormônio sexual não diferiram entre os grupos. Entretanto, reforçam que suplementação com vitamina D foi associada a maiores diminuições nos hormônios sexuais durante a perda de peso, com um possível efeito dependente da dose.

Embora os níveis séricos do micronutriente tenham aumentado após a suplementação, os autore observaram que o equilíbrio postural e os níveis de vitamina D não foram associados. Entretanto, destacam uma associação independente entre sua concentração, a força e função, uma vez que o nutriente pode contribuir de maneira indireta, regulando o sistema musculoesquelético, em especial a força muscular e a coordenação neuromuscular. 


\begin{tabular}{lrl}
\hline Serum 25-hydroxyvitamin & D & Aferir a associação das concentrações séricas \\
concentrations and lung cancer risk in & de 25 hidroxivitamina D [25 $(\mathrm{OH}) \mathrm{D}]$ com risco \\
never-smoking postmenopausal & de câncer de pulmão entre mulheres que nunca \\
women./ Cheng et al., 2017. & fumaram.
\end{tabular}

The effect of high-dose vitamin D Analisar os efeitos da suplementação com altas supplementation on muscular function doses vitamina $\mathrm{D}$, em comparação com a dose and quality of life in postmenopausal padrão, nos parâmetros de força muscular, women: A randomized controlled trial./ equilíbrio e qualidade de vida. Além dos Grimnes; Emaus; Cashman \& Jorde, possíveis efeitos musculares de polimorfismos 2017.

de nucleotídeo único (SNPs) em genes que codificam enzimas relacionadas à vitamina $\mathrm{D}$.

High dose vitamin d may improve lower urinary tract symptoms in postmenopausal women. / Oberg et al. 2017.

estudo avaliou se uma suplementação com altas doses de vitamina D afetaria o trato urinário de mulheres na menopausa, em comparação com a dose padrão.

Calcium plus vitamin D Avaliou-se o efeito da suplementação de cálcio supplementation and lung cancer e vitamina D na incidência de câncer de pulmão incidence among postmenopausal e se a proporção da ingestão de cálcio e women in the Women's Health magnésio $\left(\mathrm{Ca}^{2+}: \mathrm{Mg}^{+}\right)$modifica a associação da Initiative./Tao et al., 2017.

The effects of single high-dose or daily lowdosage oral colecalciferol treatment on vitamin D levels and muscle strength in postmenopausal women./Apaydin et al., 2018 suplementação entre os dois nutrientes $(\mathrm{CaD})$.
Comparar os efeitos de uma alta dose (única) de colecalciferol oral com uma baixa dose nos níveis de $25(\mathrm{OH}) \mathrm{D}$ e na força muscular de mulheres na pós-menopausa, com deficiência ou insuficiência de vitamina $\mathrm{D}$.
Embora não significativo, verificou-se que houve uma tendência para um risco menor da ocorrência de câncer de pulmão em mulheres com concentrações séricas de $25(\mathrm{OH}) \mathrm{D}$ mais altas e que receberam a suplementação com vitamina $\mathrm{D}$, quando comparadas com aquelas com concentrações séricas mais baixas e que não receberam a suplementação.

Em um estudo duplo cego, randomizado, 275 mulheres foram submetidas a suplementação com vitamina $\mathrm{D}$ e avaliadas quanto aos parâmetros estabelecidos, antes e após uma intervenção de 12 meses. Ao final do estudo, os autores observaram um aumento significativo nos níveis do nutriente, nas participantes que receberam altas-doses; enquanto que as que receberam a dose padrão, verificou-se um aumento moderado. Foi verificado a presença de um polimorfismo em rs3829251, e quanto aos parâmetros avaliados, não houve diferença significativa entre os grupos. Assim, destacam que os resultados não forneceram suporte para a utilização de altas doses de vitamina $\mathrm{D}$, em indivíduos já repletos de vitamina $\mathrm{D}$ em termos de melhoria da força muscular, equilíbrio ou qualidade de vida.

Após 12 meses de intervenção, os autores observaram que não houveram mudanças expressivas quanto ao perfil de sintomas do trato urinário das 297 mulheres que compuseram a amostra do estudo. Entretanto, uma redução significativa do índice de retenção urinária, foi verificada no grupo que recebeu altas doses de vitamina D3, quando comparada ao grupo de mulheres que recebeu a dose padrão.

Os autores observaram que durante todo o período de acompanhamento, a suplementação de cálcio e vitamina D não promoveu nenhuma redução na incidência de câncer de pulmão entre as mulheres na pós-menopausa. Contudo, observou-se uma associação entre a ingestão de $\mathrm{Ca}^{2+}: \mathrm{Mg}^{+}$, entre mulheres fumantes incluídas no estudo.

Os autores observaram que a quantidade de vitamina D sérica aumentou a partir da quarta semana de intervenção, perpetuando ao longo de 12 meses. Esse aumento apresentou-se de maneira mais expressiva no grupo que recebeu uma única dose. O parâmetro de força muscular apresentou melhores resultados no grupo que recebeu doses diárias. Assim, concluem que embora uma suplementação diária seja mais eficaz para melhorar a força muscular, uma única dose é efetiva para promover o aumento dos 
The effect of vitamin D3 Avaliar o efeito da normalização do status da supplementation on markers of vitamina $\mathrm{D}$ e/ou redução dos níveis de cardiovascular health in paratormônio (PTH) na atividade do sistema hyperparathyroid, vitamin $\quad \mathrm{D}$ renina-angiotensina-aldosterona e outros insufficient women: a randomized marcadores de saúde cardiovascular. placebo-controlled trial./Bislev et al. 2018.

Effects of Vitamin D3 Supplementation on Muscle Strength, Mass, and Physical Performance in Women with Vitamin D Insufficiency: A Randomized PlaceboControlled Trial./Bislev et al. 2018.

Isolated vitamin D supplementation improves the immuneinflammatory biomarkers in younger postmenopausal women: a randomized, double-blind, placebo-controlled trial. Bueloni-Dias et al., 2018.

Analisar os efeitos da suplementação de vitamina D3 na força muscular, desempenho físico e postural, bem estar e qualidade de vida, em mulheres pós-menopáusicas saudáveis.

Verificar o efeito da suplementação de vitamina 3 3 nos marcadores inflamatórios, em mulheres
Com uma suplementação de $70 \mu \mathrm{g} /$ dia de vitamina D3, por 12 meses, o autores verificaram uma elevação significativa nas concentrações de $25(\mathrm{OH}) \mathrm{D}$ e uma redução de $17 \%$ do hiperparatireoidismo secundário, observado nas mulheres pós-menopáusicas. Dentre os demais marcadores cardíacos avaliados, tais como hemoglobina glicada, pressão arterial, rigidez vascular, frequência cardíaca e condutividade cardíaca, observou-se uma alteração somente nos níveis plasmáticos de lipoproteínas de alta densidade (HDL), os quais apresentaram-se elevados.

Verificou-se um aumento nos níveis de vitamina D e uma redução nos níveis de paratormônio, em 17\%. Comparando-se ao grupo placebo, observou-se que A suplementação de vitamina D3 não alterou a produção de força máxima, bem como o perfil de atividade física, massa magra corporal total, índice de massa magra apendicular, estabilidade postural, bem-estar e qualidade de vida. Portanto, os autore sugerem um uso cauteloso de doses diárias relativamente altas de vitamina D3 no tratamento da insuficiência de vitamina $\mathrm{D}$ jovens pós-menopáusicas.

Após nove meses de tratamento com suplementação de vitamina $\mathrm{D}$, os autores constataram um aumento nos níveis de $25(\mathrm{OH}) \mathrm{D}$, acompanhado por uma redução dos níveis de citocinas envolvidas no processo inflamatório, tais como IL-5, IL-12p70, IL-17a, TNF- $\alpha$ e IFN- $\gamma$. A partir de tais achados os autores concluem que a suplementação isolada com 1000 UI de vitamina D3, em mulheres jovens na pósmenopausa, pode reduzir os biomarcadores pró-inflamatórios. 


\section{Effect of isolated vitamin D} supplementation on bone turnover markers in younger postmenopausal women: a randomized, double-blind, placebo-controlled trial./ Nahas-Neto et al., 2018.

The effect of vitamin D2 supplementation on muscle strength in early postmenopausal women: a randomized, double-blind, placebocontrolled trial./ Suebthawinkul et al., 2018.

Effects of sunlight exposure and vitamin D supplementation on vitamin D levels in postmenopausal women in rural Thailand: A randomized controlled trial./ Watcharanon et al., 2018.

Role of vitamin D in energy and bone metabolism in postmenopausal women with type 2 diabetes mellitus: A 6month follow-up evaluation./ Ogata et al., 2018.
Avaliar o efeito da suplementação de vitamina Após nove meses de intervenção, respectivamente, foi observado um aumento e uma redução nos níveis $\mathrm{D}$, de forma isolada, nos marcadores de de $25(\mathrm{OH}) \mathrm{D}$, entre o grupo que recebeu as doses de vitamina $\mathrm{D}$ e o grupo placebo. Os níveis de remodelação óssea em mulheres pós- paratormônio diminuíram no grupo suplementado, bem como os níveis dos marcadores de reposição e menopáusicas jovens. formação óssea.
Esclarecer a correlação do metabolismo ósseo e metabolismo basal em mulheres pó menopausa, que possuem diabetes tipo 2 .
Este estudo teve como objetivo avaliar o efeito da suplementação isolada de vitamina D no perfil de risco da síndrome metabólica em
Devido a uma relação existente entre vitamina D e a função respiratória, os autores concluem que esse micronutriente é um componente importante no controle desse processo, por meio da utilização de glicose. Desta forma, entendem que vitamina $\mathrm{D}$ desempenha um papel crítico na relação positiva entre o metabolismo basal e ósseo, em mulheres pós-menopáusicas com diabetes mellitus tipo 2.
Um total de 88 mulheres com níveis $<20 \mathrm{ng} / \mathrm{mL}$ de vitamina $\mathrm{D}$, foram incluídas e divididas em dois grupos: grupo com suplementação de 40.000 UI/semana e um grupo placebo. Após 12 semanas, 70\% delas atingiram uma concentração satisfatória de vitamina $\mathrm{D}$, bem como os parâmetros de força e massa muscular aumentaram no grupo que recebeu a suplementação. Entretanto, após o período de suplementação não houve diferenças significativas nas mudanças observadas nos parâmetros de força muscular, massa muscular e na área de secção transversal muscular, entre os dois grupos.

Após 12 semanas de acompanhamento, observou-se uma diminuição no níveis de paratormônio nos doi grupos, bem como verificou-se que grupo que recebeu a terapia combinada (suplementação com vitamina D + exposição solar) apresentou maiores níveis de $25(\mathrm{OH}) \mathrm{D}$, quando comparado ao grupo que recebeu apenas a exposição solar. Esse achado não apresentou o mesmo perfil em mulheres com mais de 60 anos de idade.

Em um grupo de 80 mulheres que receberam a suplementação com vitamina $\mathrm{D}$, os autores verificaram um aumento nos níveis sérios do micronutriente, quando comparadas ao grupo de mulheres que receberam o placebo $(n=80)$. Esse aumento foi acompanhado de uma redução significativa dos níveis de triglicerídeos, insulina e em um modelo homeostático da resistência à insulina. $\mathrm{Na}$ análise de risco 
The Effect of Education and Vitamin D Supplementation on the Achievement of Optimal Vitamin D Level in Korean Postmenopausal Women. Lee et al. 2019

Effect of calcium and vitamin D supplementation with and without collagen peptides on bone turnover in postmenopausal women
Analisar de forma retrospectiva o efeito de ações educativas sobre a exposição à luz solar $\mathrm{e}$ a suplementação adicional de vitamina $\mathrm{D}$, para atingir o nível ideal desse nutriente, em mulheres coreanas pós-menopáusicas com osteoporose.

Examinar a eficácia da suplementação de cálcio, vitamina $\mathrm{D}$ com ou sem peptídeos de colágeno bioativos, em mulheres pósmenopáusicas com osteopenia, durante três meses.
Um total de 61 pacientes com osteoporose foram incluídas. Destas, 40 mulheres receberam apenas a orientação educacional sobre a exposição à luz solar por 30 minutos, diariamente. Um segundo grupo foi orientado a ter a mesma exposição e recebeu a suplementação de $1.000 \mathrm{UI}$ de vitamina D/dia. No início do estudo, das 61 pacientes, 18 apresentaram deficiência de vitamina D, 25 insuficiência e 18 apresentaram níveis suficientes. Após a intervenção, nas mulheres do primeiro grupo, observou-se um aumento expressivo nas concentrações de $25(\mathrm{OH})$ D naquelas com deficiência e insuficiência dos nutriente. $\mathrm{O}$ grupo que recebeu as duas alternativas terapêuticas teve um aumento médio de 31,73 $\mathrm{ng} / \mathrm{mL}$.

Avaliando alterações em marcadores específicos da remodelação óssea (propeptídeo $\mathrm{N}$-terminal de procolágeno tipo I-P1NP e telopeptídeo C-terminal de colágeno I-CTX) no grupo que recebeu suplementação com $\mathrm{Ca}$ e vitamina $\mathrm{D}$, juntamente com o peptídeos, os autores observaram uma diminuição significativa de $13,1 \%$ e $11,4 \%$, respectivamente. No grupo que recebeu apenas a $\mathrm{Ca}^{+}$e Vitamina $\mathrm{D}$, não houve mudanças em tais marcadores.
Effect of Vitamin D Supplement on Vulvovaginal Atrophy of the Menopause. / Kamronrithisorn et al., 2020
Avaliar o efeito da suplementação oral de Comparando os efeitos da suplementação entre um grupo que recebeu vitamina $\mathrm{D}$ por semana e o grupo vitamina D na atrofia vulvovaginal em mulheres na pós-menopausa. placebo, os autores observaram uma diferença média no índice de maturação vaginal, no grupo de vitamina $\mathrm{D}$, entre o início e após seis semanas. Além disso, o pH vaginal médio e a escalva visua analógica das pacientes incluídas suplementado, melhoraram significativamente em seis e 12 semanas após o tratamento, em comparação com início da investigação. 
Como apresentado na Tabela 2, os estudos incluídos nesta revisão bibliográfica, buscaram avaliar os efeitos da suplementação de vitamina D nos mais diferentes cenários. Mulheres pós-menopáusicas foram avaliadas quanto a tal terapia, considerando a prática de atividade física (Nolasco et al., 2016), o perfil sérico de hormônios sexuais (Mason et al., 2016), o risco de câncer em fumantes (Cheng et al., 2017; Tao et al., 2017), bem como aos parâmetros musculares (Brech et al., 2017; Grimnes; Emaus; Cashman \& Jorde, 2017; Tao et al., 2017; Bislev et al. 2018; Apaydin et al., 2018) e inflamatórios (BueloniDias et al., 2018).

Considerando a gravidade da deficiência de vitamina D como um problema de saúde pública, principalmente em mulheres na menopausa, compreende-se a importância de cada estudo desenvolvido a fim de se avaliar os efeitos da suplementação isolada (Cangussu et al., 2016; Bueloni-Dias et al., 2018; Nahas-Neto et al., 2018) ou combinada (Tao et al., 2017; Boursiquot et al., 2018; Watcharanon et al., 2018). Adicionalmente, destaca-se a realização de estudos que visaram compreender a relação da complexidade do quadro na promoção de alterações no trato urinário (Oberg et al. 2017; Kamronrithisorn et al., 2020) e cardiovascular (Bislev et al. 2018; Boursiquot et al., 2018). Outra característica avaliada entre os estudos incluídos foi a comparação entre a suplementação com altas e baixas doses, ou o uso de doses padrão, de vitamina D (Grimnes; Emaus; Cashman \& Jorde, 2017; Oberg et al. 2017).

Ao avaliar os tipos de estudos desenvolvidos sobre o tema, foi possível perceber um número expressivo de estudos clínicos randomizados. Esse tipo de estudo é definido pela literatura como um importante modelo investigativo, por oferecer maiores evidência acerca da análise comparada com um grupo placebo (Oliveira \& Parente, 2010; Oliveira; Velarde \& Sá, 2015). Embora permitam uma análise mais evidente, ressalta-se que nem todos os estudos aqui incluídos, apresentaram resultados com diferenças estatisticamente significativas entre os grupos, o que demonstra a necessidade de estudos contínuos para reavaliar as referidas abordagens.

A exemplo disso, Mason et al. (2016) comparou os efeitos da administração de vitamina D nos hormônios sexuais de mulheres pós-menopáusicas, que praticaram exercício para perda de peso, com o grupo placebo, que não recebeu suplementação. Os autores ressaltam que não houve alterações significativas nos níveis globulina de ligação aos hormônios sexuais, estrona, estradiol total, testosterona livre e biodisponível. Para eles, as relações entre a vitamina D e os hormônios esteróides sexuais, justificam uma investigação mais aprofundada em estudos cuidadosamente projetados para elucidar mais completamente seu papel na pós-menopausa.

Já no estudo de Nolasco et al. (2016), as participantes foram divididas em três grupos: um grupo controle sem vitamina $\mathrm{D}$ e suplementação de cálcio e que não praticou atividade física, um grupo que recebeu a suplementação com tais nutrientes e não fez exercício físico e um grupo que realizou exercícios aquáticos três vezes por semana e recebeu suplementação de vitamina D e cálcio. A partir dessa distribuição interna de grupos, entende-se que os autores desses estudos podem estabelecer comparações entre tais, analisando com maior atenção as particularidades obtidas entre cada um deles. Assim, é possível verificar, com mais evidências, os efeitos das intervenções realizadas.

Os músculos esqueléticos humanos expressam receptores de vitamina $\mathrm{D}$, e sabe-se que este nutriente tem papel importante na força e função muscular. A força muscular pode desempenhar um papel patogênico nas quedas e/ou fraturas, que são eventos muito comuns em mulheres na menopausa (Rejnmark, 2011). Neste sentido, observou que alguns estudos tiveram por finalidade avaliar os efeitos da suplementação com vitamina D, em parâmetros musculares, quando administrada em altas e baixas doses. No estudo de Grimnes, Emaus, Cashman \& Jorde (2017), o tratamento de um ano com altas doses de vitamina D não teve efeito na força muscular, equilíbrio ou qualidade de vida em mulheres pós-menopáusicas com osteopenia ou osteoporose em comparação com a dose padrão.

De maneira semelhante, Apaydin et al. (2018) incluíram em seu estudo um total de 60 mulheres, divididas em dois grupos: um grupo recebeu doses diárias de vitamina D3 e o outro grupo que recebeu uma única dose elevada do nutriente. 
Tanto os níveis séricos de vitamina D como a força muscular feminina, foram avaliados no início do estudo e após 12 meses de acompanhamento. Nisso, os autores verificaram que a quantidade de vitamina D sérica aumentou a partir da quarta semana de intervenção, e que esse aumento foi mais expressivo no grupo que recebeu uma única dose. No entanto, concluíram que embora uma dose única seja efetiva para promover o aumento dos níveis de vitamina $\mathrm{D}$, a suplementação diária é mais eficaz para melhorar a força muscular.

O estudo de Bislev et al. (2018) analisou os efeitos da suplementação em parâmetros musculares e na qualidade de vida de mulheres pós-menopáusicas com níveis plasmáticos $25(\mathrm{OH})$ D abaixo de $<50 \mathrm{nmol} / \mathrm{l}$ e níveis elevados de hormônio da paratireóide (PTH). A suplementação promoveu um aumento nos níveis de vitamina D e diminuiu os níveis de paratormônio. Essa mudança nos níveis do micronutriente não modificou nenhum parâmetro de força muscular ou qualidade de vida, de maneira que os autores sugerem o uso cauteloso de altas doses de vitamina D3. Suebthawinkul et al. (2018) obtiveram resultados semelhantes ao verificarem que após o período de suplementação, não houve diferenças significativas nas mudanças observadas nos parâmetros de força muscular, massa muscular e na área de secção transversal muscular, entre o grupo teste e o grupo placebo.

Outros aspectos também foram avaliados, como por exemplo no estudo de Oberg et al. (2017), onde o comparativo entre doses mais elevadas e dose padrão foi realizado para avaliar os efeitos dessas administrações nos sintomas de trato urinário. Um total de 297 mulheres com um perfil de densidade mineral óssea reduzida, foram incluídas no estudo. Estas receberam uma elevada dose de vitamina D3, duas vezes por semana; outro grupo recebeu uma dose padrão. Após 12 meses, os autores observaram que não houveram mudanças expressivas quanto ao perfil de sintomas do trato urinário das participantes. Entretanto, uma redução significativa do índice de retenção urinária, foi verificada no grupo que recebeu altas doses. Embora o estudo apresente limitações, os autores reportam que os resultados sugerem um possível papel da vitamina D na melhoria dos sintomas de trato urinário em mulheres pós-menopáusicas.

Ainda com uma abordagem voltada ao aspecto do trato urinário, Kamronrithisorn et al. (2020), verificou que a suplementação oral de vitamina D melhora potencialmente os resultados de saúde vaginal em mulheres na pós-menopausa com sintomas de atrofia vulvovagional, demonstrado pela melhora do índice médio de maturação vaginal, $\mathrm{pH}$ vaginal e escalva visual analógica, entre seis e 12 semanas após início da suplementação.

Considerando que a deficiência de vitamina D está relacionada com a ocorrência de outros problemas de saúde como o câncer, dois estudos incluídos nesta revisão bibliográfica avaliaram os efeitos da suplementação com esse micronutriente nos riscos de desenvolvimento do câncer de pulmão. Cheng et al. (2017) observaram que ao avaliarem mulheres pós-menopáusicas que nunca fumaram, os dados obtidos na investigação não apoiaram a hipótese de que concentrações séricas de $25(\mathrm{OH}) \mathrm{D}$ mais altas, quando comparada a concentrações mais baixas, estejam associadas a um risco menor para o desenvolvimento da doença.

Por outro lado, Tao et al. (2017) viram que suplementação de cálcio e vitamina D não promoveu nenhuma redução na incidência de câncer de pulmão entre as mulheres na pós-menopausa. A abordagem investigada por tais estudos denota a importância de compreender o cenário da ocorrência de outros problemas de saúde pública como reflexo da deficiência de vitamina D na população em estudo. Pesquisas como estas podem abrir novos caminhos para a realização de outros estudos, a fim de entender os mecanismos fisiopatológicos envolvidos.

Uma vez que efeitos cardiovasculares adversos decorrente da insuficiência de vitamina D podem ser parcialmente causados pelo desenvolvimento de hiperparatireoidismo secundário, com aumento da atividade do sistema renina-angiotensinaaldosterona (RAAS), o estudo de Bislev et al. (2018) buscou avaliar os efeitos da normalização dos níveis desse nutriente e redução do paratormônio (PTH) no sistema RAAS. O tratamento com vitamina D3 reduziu o PTH em 17\%, mas não reduziu a 
atividade do RAAS. Em comparação com o placebo, a suplementação com vitamina D3 aumentou os níveis plasmáticos de lipoproteínas de alta densidade (HDL) em 4,6\%, mas não afetou outros índices cardiovasculares.

Também em um sentido preventivo, Ferreira et al. (2019) avaliou o efeito da suplementação com 1000 UI de vitamina D3/dia na redução do risco de síndrome metabólica em mulheres na pós-menopausa com deficiência desse nutriente. As mulheres que foram submetidas à suplementação isolada com vitamina $\mathrm{D}$, por 9 meses, tiveram menor risco desenvolver a síndrome metabólica, hipertrigliceridemia e hiperglicemia.

É possível perceber que os estudos supracitados demonstram uma preocupação em compreender os efeitos da suplementação com vitamina D no desenvolvimento de outros problemas de saúde, como câncer e problema cardiovasculares, os quais são apontados como importantes doenças agudas e crônicas comuns em mulheres que apresentam deficiência desse nutriente (Pérez-López et al., 2012; Lerchbaum, 2014; Pérez-López; Chedraui; Pilz, 2020).

A exposição à luz solar é uma importante fonte de vitamina D. Nesse sentindo, os estudos de Watcharanon et al. (2018) e Lee et al (2019), associaram essa terapia à suplementação com vitamina D. No estudo de Watcharanon et al. (2018), constatou-se que a terapia combinada de uma suplementação semanal de vitamina D2 com exposição ao sol, é mais eficaz do que a exposição a luz solar sozinha, em mulheres pós-menopáusicas tailandesas. A exposição à luz solar por si só não é suficiente para manter os níveis de 25-hidroxivitamina D neste ambiente. Na investigação de Lee et al. (2019), os autores instruíram um grupo de participantes, de maneira educativa, à realizarem uma exposição solar diariamente, durante 30 minutos. Outro grupo recebeu a mesma orientação, bem como a suplementação com o nutriente. Nisso, verificaram que a maioria delas $(95 \%)$ tem potencial para atingir níveis ideais, após intervenção educacional e suplementação adicional (1.800$2.000 \mathrm{UI})$.

Ainda em ações combinadas com outros elementos, Argyrou et al. (2020) verificou que a adição de peptídeos de colágenos na suplementação de cálcio e vitamina $\mathrm{D}$, pode aumentar seu já conhecido efeito positivo no metabolismo ósseo. Essa conclusão foi alcançada através de mudanças nos níveis de importantes marcadores de formação e reabsorção óssea, como propeptídeo N-terminal de procolágeno tipo I (P1NP) e telopeptídeo C-terminal de colágeno I (CTX), respectivamente.

Analisando as publicações, percebe-se uma forte presença de estudos que avaliaram a suplementação combinada de vitamina $\mathrm{D}$, seja com outros elementos nutricionais, tais como cálcio, seja pela implementação de atividade física e/ou exposição à luz solar. Isso demonstra que muitos pesquisadores encaram a combinação terapêutica, como ações mais promissoras ao tratamento da deficiência de vitamina D em mulheres pós-menopáusicas. Adicionalmente, vale ressaltar a gama de aspectos avaliados nesse contexto, o que denota a amplitude desse problema de saúde pública entre essa população.

\section{Conclusão}

A partir do levantamento realizado percebe-se que no período de 2016 a 2020, diferentes estudos científicos foram desenvolvidos acerca da suplementação com vitamina D em mulheres pós-menopáusicas. Adicionalmente, verificou-se que a suplementação com esse nutriente é um tema amplamente avaliado, constando-se que a complexidade da ausência do mesmo é alvo de muitos pesquisadores, os quais buscaram avaliar os efeitos de tal suplementação nos mais diferentes aspectos.

Nesse sentido, destaca-se as investigações conduzidas a fim de verificar os efeitos na força muscular e remodelação óssea, uma vez que a vitamina D participa desse processo diminuindo os riscos de quedas e fraturas, comuns em mulheres na menopausa. Foi observado ainda que a suplementação combinada foi a abordagem mais desenvolvida entre os estudos, sendo esta analisada tanto com participação de outros nutrientes como também com outras ações terapêuticas como a luz solar.

A partir da reflexão apresentada no presente estudo, foi possível compreender que a suplementação exógena com vitamina D é uma opção terapêutica em constante avaliação. Assim, entende-se que mais estudos são necessários para entender o real efeito desta adição na qualidade de vida de mulheres pós-menopáusicas que apresentam deficiência do nutriente. Diante 
do exposto, acredita-se que a realização de novos estudos, como a presente revisão de literatura, pode colaborar para uma atualização contínua sobre o tema.

\section{Referências}

Apaydin, M., Can, A. G., Kizilgul, M., Beysel, S., Kan, S., Caliskan, M., \& Cakal, E. (2018). The effects of single high-dose or daily low-dosage oral colecalciferol treatment on vitamin $\mathrm{D}$ levels and muscle strength in postmenopausal women. BMC endocrine disorders, 18(1), 1-8. https://bmcendocrdisord.biomedcentral.com/articles/10.1186/s12902-018-0277-8.

Argyrou, C., Karlafti, E., Lampropoulou-Adamidou, K., Tournis, S., Makris, K., Trovas, G., \& Triantafyllopoulos, I. K. (2020). Effect of calcium and vitamin D supplementation with and without collagen peptides on bone turnover in postmenopausal women with osteopenia. Journal of musculoskeletal \& neuronal interactions, 20(1), 12. https://www.ncbi.nlm.nih.gov/pmc/articles/PMC7104583/.

Barral, D., Barros, A. C., \& de ARAÚJO, R. P. C. (2007). Vitamina D: uma abordagem molecular. Pesquisa Brasileira em Odontopediatria e Clínica Integrada, 7(3), 309-315. https://www.redalyc.org/pdf/637/63770319.pdf.

Bislev, L. S., Rødbro, L. L., Bech, J. N., Pedersen, E. B., Kjaergaard, A. D., Ladefoged, S. A., \& Rejnmark, L. (2018). The effect of vitamin D3 supplementation on markers of cardiovascular health in hyperparathyroid, vitamin $\mathrm{D}$ insufficient women: a randomized placebo-controlled trial. Endocrine, 62(1), 182-194. https://link.springer.com/article/10.1007/s12020-018-1659-4.

Bislev, L. S., Rødbro, L. L., Rolighed, L., Sikjaer, T., \& Rejnmark, L. (2018). Effects of vitamin D3 supplementation on muscle strength, mass, and physical performance in women with vitamin D insufficiency: a randomized placebo-controlled trial. Calcified tissue international, 103(5), 483-493. https://link.springer.com/article/10.1007/s00223-018-0443-z.

Boursiquot, B. C., Larson, J. C., Shalash, O. A., Vitolins, M. Z., Soliman, E. Z., \& Perez, M. V. (2019). Vitamin D with calcium supplementation and risk of atrial fibrillation in postmenopausal women. American heart journal, 209, 68-78. https://www.sciencedirect.com/science/article/abs/pii/S0002870318303454.

Brasil. Ministério da Saúde (2016). Protocolos da Atenção Básica: Saúde das Mulheres / Ministério da Saúde, Instituto Sírio-Libanês de Ensino e Pesquisa Brasília: Ministério da Saúde. http://bvsms.saude.gov.br/bvs/publicacoes/protocolos_atencao_basica_saude_mulheres.pdf.

Brech, G. C., Ciolac, E. G., Peterson, M. D., \& Greve, J. M. D. A. (2017). Serum 25-hydroxyvitamin D levels are associated with functional capacity but not with postural balance in osteoporotic postmenopausal women. Clinics, 72(1), 11-16. https://www.scielo.br/scielo.php?script=sci_arttext\&pid=S180759322017000100011.

Bueloni-Dias, F. N., Orsatti, C. L., Cangussu, L. M., Poloni, P. F., Spadoto-Dias, D., Nahas-Neto, J., \& Nahas, E. A. (2018). Isolated vitamin D supplementation improves the immune-inflammatory biomarkers in younger postmenopausal women: a randomized, double-blind, placebo-controlled trial. Menopause, 25(8),

https://journals.lww.com/menopausejournal/Abstract/2018/08000/Isolated_vitamin_D_supplementation_improves_the.9.aspx.

$897-903$.

Cangussu, L. M., Nahas-Neto, J., Orsatti, C. L., Poloni, P. F., Schmitt, E. B., Almeida-Filho, B., \& Nahas, E. A. P. (2016). Effect of isolated vitamin D supplementation on the rate of falls and postural balance in postmenopausal women fallers: a randomized, double-blind, placebo-controlled trial. Menopause, 23(3), 267-274. https://journals.lww.com/menopausejournal/Abstract/2016/03000/Effect_of_isolated_vitamin_D_sup plementation_on.8.aspx.

Castro, L. C. G. D. (2011). O sistema endocrinológico vitamina D. Arquivos Brasileiros de Endocrinologia \& Metabologia,55(8), 566-575. https://www.scielo.br/scielo.php?pid=S0004-27302011000800010\&script=sci_arttext\&tlng=pt.

Cheng, T. Y. D., Song, X., Beresford, S. A., Ho, G. Y., Johnson, K. C., Datta, M., \& Neuhouser, M. L. (2017). Serum 25-hydroxyvitamin D concentrations and lung cancer risk in never-smoking postmenopausal women. Cancer Causes \& Control,28(10), 1053-1063. https://link.springer.com/article/10.1007/s10552-017-0956-1.

El Khoudary, S. R., Greendale, G., Crawford, S. L., Avis, N. E., Brooks, M. M., Thurston, R. C., \& Matthews, K. (2019). The menopause transition and women's health at midlife: a progress report from the Study of Women's Health Across the Nation (SWAN). Menopause (New York, NY), 26(10), 1213. https://www.ncbi.nlm.nih.gov/pmc/articles/PMC6784846/.

Ercole, F. F., Melo, L. S., Alcoforado, C. L. G. C. (2014). Revisão integrativa versus revisão sistemática. Revista Mineira de Enfermagem, 18(1), 9-12. http://www.reme.org.br/artigo/detalhes/904.

Ferreira, P. P., Cangussu, L., Bueloni-Dias, F. N., Orsatti, C. L., Schmitt, E. B., Nahas-Neto, J., \& Nahas, E. A. P. (2020). Vitamin D supplementation improves the metabolic syndrome risk profile in postmenopausal women. Climacteric, 23(1), 24-31. https://www.tandfonline.com/doi/abs/10.1080/13697137.2019.1611761.

Grimnes, G., Emaus, N., Cashman, KD, \& Jorde, R. (2017). O efeito da suplementação de vitamina D em altas doses na função muscular e na qualidade de vida em mulheres na pós-menopausa - um ensaio clínico randomizado. Clinical endocrinology, 87 (1), 20-28. https://onlinelibrary.wiley.com/doi/abs/10.1111/cen.13353.

Harlow, S. D., Gass, M., Hall, J. E., Lobo, R., Maki, P., Rebar, R. W., \& STRAW+ 10 Collaborative Group. (2012). Executive summary of the Stages of Reproductive Aging Workshop+ 10: addressing the unfinished agenda of staging reproductive aging. The Journal of Clinical Endocrinology \& Metabolism, 97(4), 1159-1168. https://academic.oup.com/jcem/article/97/4/1159/2833227?login=true.

Kamronrithisorn, T., Manonai, J., Vallibhakara, SAO, Sophonsritsuk, A., \& Vallibhakara, O. (2020). Efeito do suplemento de vitamina D na atrofia vulvovaginal da menopausa. Nutrients, 12 (9), 2876. https://www.mdpi.com/2072-6643/12/9/2876. 
Lee, C. J., Kim, S. S., Suh, W. Y., Kim, J. S., Jung, J. G., Yoon, S. J., \& Yang, H. J. (2019). The Effect of Education and Vitamin D Supplementation on the Achievement of Optimal Vitamin D Level in Korean Postmenopausal Women. Journal of bone metabolism,26(3), 193. https://synapse.koreamed.org/upload/SynapseData/PDFData/2187JBM/jbm-26-193.pdf.

Lee, J. S., \& Kim, J. W. (2018). Prevalence of vitamin D deficiency in postmenopausal high-and low-energy fracture patient. Archives of osteoporosis, 13(1), 1-6. https://link.springer.com/article/10.1007/s11657-018-0524-7.

Lerchbaum, E. (2014). Vitamin D and menopause-A narrative review. Maturitas, 79(1), https://www.sciencedirect.com/science/article/abs/pii/S0378512214001996.

Lui Filho, J. F., Baccaro, L. F. C., Fernandes, T., Conde, D. M., Costa-Paiva, L., \& Pinto Neto, A. M. (2015). Epidemiologia da menopausa e dos sintomas climatéricos em mulheres de uma região metropolitana no sudeste do Brasil: inquérito populacional domiciliar. Revista Brasileira de Ginecologia e Obstetrícia, 37(4), 152-158. https://www.scielo.br/scielo.php?pid=S0100-72032015000400152\&script=sci_arttext.

Mason, C., Tapsoba, J. D. D., Duggan, C., Imayama, I., Wang, C. Y., Korde, L. A., \& McTiernan, A. (2016). Effects of vitamin D supplementation during weight loss on sex hormones in postmenopausal women. Menopause (New York, NY), 23(6), 645. https://www.ncbi.nlm.nih.gov/pmc/articles/PMC4874908/.

Monteleone, P., Mascagni, G., Giannini, A., Genazzani, A. R., \& Simoncini, T. (2018). Symptoms of menopause-global prevalence, physiology and implications. Nature Reviews Endocrinology, 14(4), 199. https://www.nature.com/articles/nrendo.2017.180.

Nahas-Neto, J., Cangussu, L. M., Orsatti, C. L., Bueloni-Dias, F. N., Poloni, P. F., Schmitt, E. B., \& Nahas, E. A. P. (2018). Effect of isolated vitamin D supplementation on bone turnover markers in younger postmenopausal women: a randomized, double-blind, placebo-controlled trial. Osteoporosis International, 29(5), 1125-1133. https://link.springer.com/article/10.1007/s00198-018-4395-y.

National Collaborating Centre for Women's and Children's Health (Reino Unido. (2015). Menopause: Full Guideline. https://pubmed.ncbi.nlm.nih.gov/26598775/.

Nolasco, R., Moreira, L. D., Bocalini, D. S., Fronza, F. C., Marin, R. V., \& Lazaretti-Castro, M. (2017). Effects of vitamin D supplementation on pulmonary function in postmenopausal women following an aquatic exercise program. Archives of endocrinology and metabolism,61(1), 28-35. https://www.scielo.br/scielo.php?pid=S2359-39972016005005105\&script=sci_arttext.

Oberg, J., Verelst, M., Jorde, R., Cashman, K., \& Grimnes, G. (2017). High dose vitamin D may improve lower urinary tract symptoms in postmenopausal women. The Journal of steroid biochemistry and molecular biology, 173, 28-32. https://www.sciencedirect.com/science/article/abs/pii/S096007601730078X.

Ogata, M., Iwasaki, N., Ide, R., Takizawa, M., Tanaka, M., Tetsuo, T., \& Uchigata, Y. (2018). Role of vitamin D in energy and bone metabolism in postmenopausal women with type 2 diabetes mellitus: A 6-month follow-up evaluation. Journal of diabetes investigation,9(1), 211-222. https://onlinelibrary.wiley.com/doi/full/10.1111/jdi.12666.

Oliveri, B., Mastaglia, S. R., Brito, G. M., Seijo, M., Keller, G. A., Somoza, J., \& Di Girolamo, G. (2015). Vitamin D3 seems more appropriate than D2 to sustain adequate levels of 25OHD: a pharmacokinetic approach. European journal of clinical nutrition,69(6), 697-702. https://www.nature.com/articles/ejcn201516?page=4\&page $=2$.

Pérez-López, F. R., Brincat, M., Erel, C. T., Tremollieres, F., Gambacciani, M., Lambrinoudaki, I., \& Rees, M. (2012). EMAS position statement: vitamin D and postmenopausal health. Maturitas, 71(1), 83-88. https://www.sciencedirect.com/science/article/abs/pii/S0378512211003653.

Pérez-López, F. R., Chedraui, P., \& Pilz, S. (2020). Vitamin D supplementation after the menopause. Therapeutic Advances in Endocrinology and Metabolism, 11, 2042018820931291. https://journals.sagepub.com/doi/full/10.1177/2042018820931291.

Potter, B.; Schrager, S.; Dalby, J.; Torell, E.; Hampton, A. (2018). Menopause. Primary Care: Clinics in Office Practice 45(4), 625-641. https://www.sciencedirect.com/science/article/abs/pii/S0095454318300745?via\%3Dihub

Prodanov, C. C., \& de Freitas, E. C. (2013). Metodologia do trabalho científico: métodos e técnicas da pesquisa e do trabalho acadêmico. Editora Feevale.

Rejnmark, L. (2011). Efeitos da vitamina D na função e desempenho muscular: uma revisão das evidências de ensaios clínicos randomizados. Avanços terapêuticos em doenças crônicas, 2 (1), 25-37. https://journals.sagepub.com/doi/abs/10.1177/2040622310381934.

Rother, E. T. (2007). Revisão sistemática X revisão narrativa. Acta paulista de enfermagem, 20(2), v-vi. https://www.scielo.br/pdf/ape/v20n2/a01v20n2.pdf.

Schmitt, E. B., Nahas-Neto, J., Bueloni-Dias, F., Poloni, P. F., Orsatti, C. L., \& Nahas, E. A. P. (2018). Vitamin D deficiency is associated with metabolic syndrome in postmenopausal women. Maturitas, 107, 97-102. https://www.sciencedirect.com/science/article/abs/pii/S037851221730628X.

Suebthawinkul, C., Panyakhamlerd, K., Yotnuengnit, P., Suwan, A., Chaiyasit, N., \& Taechakraichana, N. (2018). O efeito da suplementação de vitamina D2 na força muscular em mulheres na pós-menopausa: um estudo randomizado, duplo-cego e controlado por placebo. Climatério, 21 (5), 491-497. https://www.tandfonline.com/doi/abs/10.1080/13697137.2018.1480600.

Tao, M. H., Dai, Q., Chen, S., Freudenheim, J. L., Rohan, T., Wakelee, H., \& Wactawski-Wende, J. (2017). Calcium plus vitamin D supplementation and lung cancer incidence among postmenopausal women in the Women's Health Initiative. Lung Cancer, 110, 42-47. https://www.sciencedirect.com/science/article/abs/pii/S0169500217303550.

Vaes, A. M., Tieland, M., de Regt, M. F., Wittwer, J., van Loon, L. J., \& de Groot, L. C. (2018). Dose-response effects of supplementation with calcifediol on serum 25-hydroxyvitamin D status and its metabolites: a randomized controlled trial in older adults. Clinical nutrition, 37(3), 808-814. https://www.sciencedirect.com/science/article/abs/pii/S0261561417301206.

Watcharanon, W., Kaewrudee, S., Soontrapa, S., Somboonporn, W., Srisaenpang, P., Panpanit, L., \& Pongchaiyakul, C. (2018). Effects of sunlight exposure and vitamin D supplementation on vitamin D levels in postmenopausal women in rural Thailand: A randomized controlled trial. Complementary therapies in medicine, 40, 243-247. https://www.sciencedirect.com/science/article/abs/pii/S0965229918302504. 